\title{
El concepto de sophrosyne en los diálogos platónicos y su ejemplificación en la figura de Sócrates
}

\author{
Carreño, Sofía \\ El concepto de sophrosyne en los diálogos platónicos y su ejemplificación en la figura de Sócrates \\ Synthesis, vol. 26, núm. 2, 2019 \\ Universidad Nacional de La Plata, Argentina \\ Disponible en: http://www.redalyc.org/articulo.oa?id=84662943006 \\ DOI: https://doi.org/10.24215/1851779Xe063
}

Esta obra está bajo una Licencia Creative Commons Atribución-NoComercial-Compartirlgual 4.0 Internacional. 


\title{
El concepto de sophrosyne en los diálogos platónicos y su ejemplificación en la figura de Sócrates
}

\author{
The Concept Sophrosyne in the Platonic Dialogues and its Depiction in Socrates' Figure. \\ Sofía Carreño \\ Universidad Nacional de Colombia, Colombia \\ scarrenoc@unal.edu.co \\ DOI: https://doi.org/10.24215/1851779Xe063 \\ Redalyc: http://www.redalyc.org/articulo.oa?id=84662943006
}

(iD) http://orcid.org/0000-0002-9931-4987

Recepción: 13 Febrero 2019

Aprobación: 06 Diciembre 2019

\begin{abstract}
Resumen:
La sensatez (sophrosýne/ $\sigma \omega \phi p o \sigma i v \eta)$ es un tema recurrente en los diálogos de Platón. Sin embargo, allí no es clara la relación de Sócrates con el conocimiento de sí. Por ende, en el presente trabajo, comenzaré por determinar la relación entre conocimiento de sí y sophrosýne para luego establecer cómo es definida la sophrosýne en los diálogos y cómo se puede llegar a poseerla. Luego, abordaré la pregunta acerca de si Sócrates puede ser calificado como sensato bajo su propia definición, pregunta que recibirá una respuesta negativa. No obstante, argumentaré que Sócrates no sólo cuenta con el conocimiento acerca de cómo alcanzar la sophrosýne, sino que además parece no haber nada que le impida hacerlo.
\end{abstract}

Palabras Clave: Sensatez, Virtud, Élenchos, Conocimiento, Moral.

\section{Abstract:}

Sanity (sophrosýne/ $\sigma \omega \phi p \circ \sigma \dot{v} \eta)$ is a recurring topic throughout the platonic dialogues. However, the relationship between Socrates and self-knowledge is not yet clear. Hence, in the following text, I will begin by determining the relationship between selfknowledge and sophrosýnein order to stablish how is the concept of sophrosíne defined in the dialogues and how can one reach it. Afterwards, I will address the question regarding Socrates' sanity according to his own definition, question that will receive a negative answer. Nonetheless, I will argue that Socrates does not only count with the knowledge necessary to achieve sophrosýne, but also, there seems to be that there is nothing that should stop him from achieving sophrosýne.

KEYWORDS: Sanity, Virtue, Élenchos, Knowledge, Morals.

La sensatez (sophrosýne/ $\sigma \omega \phi p o \sigma \dot{v} \eta)$ es un tema recurrente que atraviesa diversos diálogos de Platón, en los cuales esta virtud se presenta en relación con el éros, el conocimiento de sí, y la política. (Por ejemplo, esta virtud aparece como tema central en el Cármides, en el Laques se explora la relación entre sophrosýne y andreía/valor en 193a, el Fedro nos brinda una definición clara y concisa de la sophrosýne como dominio de sí en 238a, la República nos presenta un contraste entre la sophrosýne y la locura en 585b y define en 427e la sophrosýne en tanto virtud capital como el ocuparse de lo suyo, y en el Protágoras, entre 332a y 333b, la sophrosýne se equipara a la sophía cuando se discute la unidad de las virtudes). No obstante, en el presente trabajo me enfocaré en los diálogos tempranos (aunque también haré uso de otros diálogos donde se expone el concepto de sophrosýne), pues es allí donde vemos a un Sócrates interesado principalmente en cuestiones éticas enmarcadas dentro de la pregunta por cómo se debe vivir. Ahora bien, para llegar a ver la importancia de preguntarnos por la sophrosýne, debemos empezar por preguntarnos lo siguiente: ¿logró Sócrates resolver la pregunta acerca de cómo se debe vivir? La Apología nos sugiere que hasta cierto punto sí lo hizo, pues en este diálogo se concluye que Sócrates es el más sabio entre sus conciudadanos dado que parece cumplir la máxima délfica "conócete a ti mismo". Sin embrago, la manera en que Sócrates cumple con esta máxima es inquietante, dado que su conocimiento de sí mismo consiste en su reconocimiento del hecho de que no posee sabiduría moral alguna, lo cual lo lleva a no actuar como si fuese un sabio, a diferencia de sus conciudadanos que 
aparentan ser sabios sin serlo ni saber que no lo son. En este sentido, parece que la manera en que la máxima délfica es interpretada en este diálogo es la siguiente: el conocimiento de sí consiste en un reconocimiento de la propia ignorancia el cual lleva a vivir buscando el conocimiento que no se posee y sin aparentar ser sabio.

Sin embargo, cabe preguntarnos si es realmente esto a lo que apunta la máxima délfica al hablar de conocimiento de sí, pues decir que alguien es más sabio que otro no necesariamente quiere decir que dicho alguien es sabio, más aún en este caso donde el único conocimiento que posee Sócrates es el de su falta de sabiduría en términos morales. En este sentido, se abren varias preguntas acerca de la relación de Sócrates con la sabiduría y el conocimiento de sí: ¿En qué sentido se puede decir que Sócrates se conoce a sí mismo? ¿Es el conocimiento de sí una cuestión gradual? ¿Qué gana Sócrates con conocerse a sí mismo en mayor medida que sus conciudadanos? ¿Es el conocimiento de sí lo mismo que la sabiduría?

En el intento por responder estas interrogantes, haré uso del concepto de sophrosýne y la manera en que este es utilizado en los diálogos platónicos, y presentado con relación a la figura de Sócrates. Así, comenzaré por determinar la relación entre conocimiento de sí, sabiduría y sophrosýne para luego establecer cómo es definida la sophrosýne en los diálogos y posteriormente cómo se puede llegar a poseerla. Después de esto, abordaré la pregunta acerca de si Sócrates puede ser calificado como sensato bajo su propia definición, pregunta que recibirá una respuesta negativa. No obstante, argumentaré que Sócrates no sólo cuenta con el conocimiento acerca de cómo alcanzar la sophrosýne, sino que además parece no haber nada que le impida hacerlo.

Para cumplir con los propósitos anteriormente planteados, y teniendo en cuenta que la figura de Sócrates nos es presentada con diversos matices en los diferentes diálogos, enfocaré mi trabajo en el Sócrates del Cármides, siendo este el diálogo que más explícitamente nos habla sobre la sophrosýne. Adicionalmente, para establecer cómo es que Sócrates define la sophrosýne, utilizaré las definiciones presentadas en el Cármides, al igual que algunos pasajes del Alcibiades ${ }^{1}$ y el Fedro. Para establecer cómo se puede alcanzar la sophrosýne -si es que se puede-, me serviré de los comentarios de Rappe, Kosman y Schmid, y me remitiré tanto al Cármides como a algunos pasajes del Alcibiades I. Para evaluar si Sócrates es o no sensato bajo su propia definición, haré uso del Cármides, el Banquete y la Apología.

Antes de comenzar, cabe notar que la palabra sophrosýne, en el contexto de los diálogos platónicos, ha contado con diferentes traducciones. En el español esta ha sido principalmente traducida como "sensatez", aunque en algunos casos ( Protágoras, 323a; Burnet, 1903) se ha traducido como "moderación". En el inglés suele traducirse como "temperance" (trad. Jowett, 2008) que denota moderación y templanza, en el francés como "sagesse" (trad. Chambry, 2015) que denota sabiduría y buen juicio y en el alemán de forma sustantivada como "Besonnenheit" que denota buen juicio, sensatez y prudencia o de forma verbal como "besonnen" que adicionalmente denota serenidad (trad. Haller, 2005). Creyendo que la palabra sophrosýne abarca todas estas formas de traducción, haré uso de ella principalmente como "sensatez" al ser la forma de traducción más abarcante. Sin embrago, en algunos momentos usaré el término "moderación" si es el caso de que los interlocutores en el diálogo estén enfatizando en este matiz de la sophrosýne como ocurre en Cármides 159b. Igualmente, por momentos hablaré de la sophrosýne como dominio de sí o autocontrol cuando me refiera a los casos en que esta virtud es definida de tal manera en diálogos como el Fedro y La República.

\section{1. ¿QUÉ ES LA SOPHROSÝNE?}

\subsection{Intentos de definición}

Desde el principio del Cármides, se muestra que la búsqueda de la definición de la sophrosýne parte del presupuesto de que esta, al ser una virtud, "tiene que ser algo hermoso" ( Cármides, 160c) y por ende algo que hace buenos a quienes la posean ( Cármides, 160e). A partir de este presupuesto, la definición dada por Cármides de la sensatez como tranquilidad (Cármides, 159b) es refutada por no ser algo que necesariamente 
hace bueno a quien la posee: existen ciertas labores cuya realización exitosa depende del no ser tranquilo, la lucha, por ejemplo ( Cármides, 159c). Un argumento similar es utilizado para refutar la definición de la sensatez como pudor ( Cármides, 160e).

La definición de la sensatez - dada por Critias, quien ha sustituido a Cármides como interlocutor- como el "ocuparse de lo suyo" ( Cármides, 161e), es igualmente refutada, y pasa a ser reformulada como el "ocuparse de cosas buenas" ( Cármides, 163e). Este intento definición es en principio aceptado, pues tiene sentido que una virtud que hace del hombre bueno verse sobre las cosas buenas. Sin embargo, esta definición resulta insuficiente para Sócrates, pues, en este punto, parece que quien es sensato no necesita saber que lo es; cualquiera puede llevar a cabo una buena acción sin saber que la acción que está realizando es buena.

\subsubsection{Planteamiento de la pregunta acerca de si se debe saber que se es sensato}

La segunda parte del Cármides inicia con la afirmación hecha por Sócrates de que "parece que, quien algunas veces obra con provecho, obra, en verdad, con sensatez y es sensato, aunque no sabe que lo es" ( Cármides, 164c). Esta afirmación es inmediatamente rechazada por Critias, quien afirma:

Casi iba yo a sostener eso mismo de que ser sensato es conocerse a sí mismo, y coincido con aquel que en Delfos puso aquella inscripción que, según creo, está dedicada a esto, a una bienvenida del dios a los que entran, en lugar de decir 'salud', ya que esta fórmula de 'salud' no es correcta, ni deseable como exhortación de unos a otros, sino la de 'sé sensato'. [...] Porque el ‘conócete a ti mismo' y el 'sé sensato' son la misma cosa [...]. ( Cármides, 164e-165a)

Entonces, Sócrates se dispone a examinar esta afirmación hecha por Critias, y expone el carácter intencional de todo saber, planteando que, si la sensatez es una suerte de conocimiento, esta ha de ser conocimiento acerca de algo, ya sea un objeto concreto (como en el caso de la medicina, cuyo objeto es la salud), o un objeto abstracto (como el caso de la geometría cuyo objeto son los números).

A la pregunta por el objeto del saber que constituye la sensatez, Critias responde que la sensatez es conocimiento del conocimiento, es "el saber qué es lo que se sabe y lo que no se sabe" ( Cármides, 167a). Respuesta que resulta desconcertante, pues se esperaría que Critias contestase que uno mismo es el objeto del conocimiento constituyente de la sensatez. Sócrates intenta refutar esta definición señalando que no existen sensaciones de sí mismas, ni deseos u opiniones sin un objeto fuera de sí mismos ( Cármides, 167e). No obstante, al ver que Critias no se encuentra satisfecho con esta refutación, decide dar por sentado que se puede dar un saber del saber y procede a examinar qué pasaría si fuera el caso:

Con un saber semejante, ¿cómo sabrá [quien posea dicho saber] que conoce? Porque, a fin de cuentas, si conoce qué es la salud, es por la medicina, pero no por la sensatez, y lo armónico por la música, pero no por la sensatez, y así sucesivamente ( Cármides, 170c).

\subsubsection{Sophrosýne como conocimiento de síy del conocimiento mismo}

\subsubsection{Esbozo de la refutación}

En primer lugar, Sócrates le recuerda a Critias que conocimiento sobre el conocimiento y la ignorancia no es lo mismo que saber sobre el conocimiento o la ignorancia de algún tema específico, por ejemplo, la salud o la ley. Pues si se llega al conocimiento en áreas específicas (como la salud) a través de prácticas específicas (como la medicina), entonces ¿cómo se puede llegar al conocimiento del conocimiento mismo?

Por lo tanto, parece que quien posee el conocimiento del conocimiento, puede saber que él sabe, pero no puede saber lo que sabe. Por la misma razón, quien posea este conocimiento no podrá distinguir, por ejemplo, a un verdadero médico de alguien que dice ser médico sin serlo. Lo que le falta a este tipo de conocimiento es 
precisamente el objeto que permite distinguir una ciencia de la otra. Por ende, quien posea el conocimiento del conocimiento, no podrá, por ejemplo, tener conocimiento de una mala o buena medicina a menos que sea él mismo un médico.

Sócrates argumenta, entonces, que el conocimiento del conocimiento sería útil únicamente porque puede llevar a una visión adicional en áreas específicas del conocimiento, lo que facilita el aprendizaje concreto y la investigación ( Cármides, 172c). Sócrates procede a expresar esta idea relatando un sueño en el que existe una sociedad en la que todos sus miembros poseen el conocimiento del conocimiento. Esto llevaría a dicha sociedad a cierto grado de perfección, pues no habría nadie ocupándose de aquello sobre lo cual no tiene conocimiento, pero allí no se alcanzaría la felicidad. Esta sería alcanzada, como señala Critias, únicamente con el conocimiento acerca de lo bueno y lo malo ( Cármides, 174b). Lo cual Sócrates admite como cierto a la hora de caracterizar la sophrosýne, pero como insuficiente a la hora de definirla, pues este conocimiento de lo bueno y lo malo acompaña toda virtud.

\subsubsection{2. ¿Cómo entender la reformulación de Critias?}

Ahora bien, a pesar de haber esbozado la conversación entre Sócrates y Critias acerca de la sophrosýne como conocimiento del conocimiento, esta respuesta frente a la pregunta por el objeto de la sensatez en tanto conocimiento sigue siendo oscura y desconcertante. Frente a esto, Kosman nos indica que una posible forma de entender la respuesta de Critias es como lo haría Aristóteles: señalando que para que se pueda decir que un agente ha actuado de manera virtuosa dicho agente debe saber lo que está haciendo; debe realmente ser un agente (Kosman, 2014, pp. 227). Kosman parece indicar que lo que Critias quiere decir es que la sophrosýne como conocimiento de sí y del conocimiento mismo es i) saber lo que uno está haciendo, ii) que uno está actuando de manera sensata al saber lo que uno está haciendo, y iii) que al saber que uno está actuando de manera sensata, uno sabe que uno es sensato, pues es este saber que se sabe que se está actuando de manera sensata lo que constituye la sensatez (Kosman, 2014, pp. 229). Es tal vez en este sentido que el conocimiento del conocimiento puede ser equiparado con el conocimiento de sí, pues el conocimiento del conocimiento viene siendo un saber que se sabe algo sobre uno mismo.

Kosman también señala que este puede ser un caso de predicación Paulina: "así como decir que 'la caridad es paciente' puede significar que quienes son caritativos son pacientes [...] decir que 'el conocimiento de sí se conoce', puede significar que quien posee el conocimiento de sí, se conoce a sí mismo” (Kosman, 2014, pp. 233). ${ }^{2}$ Así, al poseer un conocimiento que se conoce a sí mismo, se será como el conocimiento que se posee, es decir, uno se conocerá a sí mismo. Esto es lo que parece afirmar Critias al decir:

Si alguno tiene un saber que se conoce a sí mismo, ese sería tal como es lo que tiene. Como cuando alguno tenga velocidad, será veloz, y cuando belleza, bello, y cuando conocimiento, conocedor, y cuando, en verdad tenga un conocimiento que lo es de sí mismo, ese tal será entonces un conocedor que se conoce a sí mismo. ( Cármides, 169e).

Esta definición es aceptada por Sócrates como coherente, y su refutación va dirigida hacia su suficiencia para hablar de la sophrosýne, pues si fuera suficiente, parecería, como ya se mostró, que la sophrosýne no posee utilidad práctica alguna. A pesar de que la refutación resulta exitosa, no hay razón para pensar que todo lo dicho por Critias deba descartarse: precisamente porque Sócrates logra demostrar la insuficiencia mas no la incoherencia de esta definición y porque, además, se acepta que, en una pequeña medida, esta definición posee cierta utilidad como acompañamiento de conocimientos prácticos y claramente intencionales ( Cármides, 172c), es que podemos pensar que aunque el conocimiento de sí como conocimiento del conocimiento no define la sophrosýne, sí es una característica de quien la posee. Ahora bien, si lo anterior es verdad, y para ser sensato se necesita conocimiento de que se es sensato, entonces, se sigue que para ser sensato se requiere saber lo que es la sensatez. 


\subsection{Sophrosýne como dominio de sí}

A pesar de que la definición de la sensatez como dominio de sí no es discutida en el Cármides, Sócrates se adhiere a ella en otros diálogos:

El hecho de que la sophrosýne se ocupa esencialmente con el autocontrol, se hace explícito en la descripción de Sócrates en la República: 'La sensatez es claramente un tipo de orden y el dominio de ciertos placeres y deseos [...]'. (Kosman, 2014,pp. 238)

Es tal vez a este tipo de definición que apuntaba Cármides al hablar de la sensatez como cierta tranquilidad o calma. Calma que vendría del no estar en conflicto con los propios deseos. Esto puede ser relacionado con la sensatez como conocimiento de sí, pues, como señala Kosman "el sensato está en control de sus acciones, es decir, sabe lo que está haciendo" (Kosman, 2014, p. 242). El hecho de que la definición de sophrosýne como tranquilidad pueda entenderse como moderación, tiene sentido a raíz del hecho de que, en su respuesta,

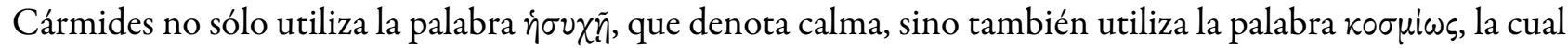
denota moderación: ${ }^{3}$

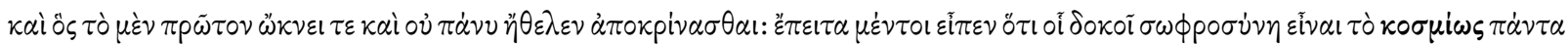

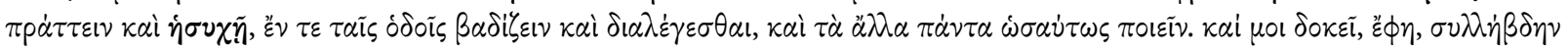

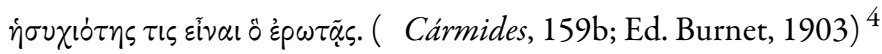

Si la sensatez es algo tuyo, tendrás una cierta opinión de ella; pues, forzosamente, estando en ti, en el supuesto de que lo esté, te suministrará una cierta sensación de la que se alce alguna posible opinión sobre ella: o sea sobre qué es y cuáles son las propiedades de la sensatez. ¿No te parece?

Para sustentar esta posible definición de sensatez, Schmid brinda cierto contexto histórico acerca del surgimiento del término sophrosýne, el cual viene de la cultura de la aristocracia doria ${ }^{5}$ y se refiere a la atención frente al comportamiento que se espera en sociedad. Tiene que ver con el control de los apetitos, y se basa en los conceptos de "honor" y "vergüenza". Además, se relaciona con el respeto hacia los demás, y el comportamiento específico que suponía el poseer la sophrosýne era dependiente del rol que se tuviese en la sociedad (Schmid, 1983, pp. 130).

Sin embargo, Schmid también señala que para el momento del Cármides, la concepción ética de la cultura doria ya se encontraba en declive, por lo cual el concepto de sophrosýne solía ser entendido como un mero control sobre los apetitos y una forma de prudencia con respecto a las relaciones interpersonales (Schmid, 1983, pp. 340-341). Es tal vez por esta razón que, si la definición de Cármides de la sensatez como tranquilidad apuntaba a una forma de autocontrol, esta no fue entendida de esta manera en el diálogo.

\subsubsection{Sophrosýne como conocimiento y dominio de si}

Ahondando en la descripción de Schmid y Kosman acerca de la sophrosýne como cierto tipo de moderación, Schmid nos dice:

La Apología cuenta la historia de su ${ }^{6}$ descubrimiento de que, aunque la gente de su tiempo había logrado un conocimiento genuino en campos de experiencia técnica, confundieron este conocimiento limitado con la sabiduría, o pensaron que también tenían un conocimiento genuino en la vida moral y política, que no tenían (21a-23 b, especialmente 22a). Así, Sócrates agregaría a los dos campos principales de aplicación de la virtud de la moderación: la conducta sexual y las relaciones interpersonales (que tenían un aspecto cívico o político), un tercer campo: la vida intelectual. (Schmid, 1983, pp. 341)

De esta forma podemos relacionar la sophrosýne como conocimiento de sí y la sophrosýne como dominio de sí. Entendiendo moderación por dominio de sí. En este sentido, en casos como el de Alcibíades en el Alcibiades $I$, vemos que una persona que cree saber qué es importante para la vida se rehúsa a cuestionar sus valores y tiene una imagen "agrandada" de sí mismo (103a-119d). Dicha persona no sólo no posee conocimiento sobre 
el objeto de sus deseos, sino tampoco posee ningún grado de conocimiento de sí al no saberse ignorante. Es esta falta de auto-conocimiento la que le impide ejercer la modestia intelectual que, como señaló Schmid, fue añadida por Sócrates a la definición clásica de sophrosýne como moderación.

\subsection{Conclusiones acerca de lo que es la sophrosýne}

Como ya era claro, el Cármides no da una definición completa de la sophrosýne y esta tampoco ha sido obtenida a partir del análisis de la definición de Critias de la sophrosýne como conocimiento de sí/ conocimiento del conocimiento, ni del contexto histórico y lo dicho en la República acerca de la sophrosýne como dominio de sí. ${ }^{7}$ No obstante, lo dicho hasta ahora permite extraer ciertas características del poseer la sophrosíne.

En primer lugar, quien posee la sophrosýne, posee un conocimiento de sí entendido como conocimiento del conocimiento, en tanto que

1. sabe lo que está haciendo;

2. sabe que al saber lo que está haciendo, está actuando de manera sensata, pues también;

3. sabe que al saber que sabe que está actuando de manera sensata, sabe que es sensato.

En otras palabras, quien posee la sophrosýne, ha de saber que sabe que es sensato al saber que sabe que está actuando de manera sensata. De lo cual se sigue que quien es sensato, ha de saber lo que es la sensatez. Esto es coherente con lo que sostiene Sócrates al principio de Cármides al decirle a Cármides que "es manifiesto que, si la sensatez es algo tuyo, tendrás una cierta opinión de ella [...]”. ( Cármides, 159a)

En segundo lugar, quien posee la sophrosýne, ha de poseer un conocimiento acerca de lo bueno y lo malo, es decir, ha de poseer cierto conocimiento moral.

Además, teniendo en cuenta el contexto histórico del término sophrosýne, y la imagen de sí que tiene quien no la posee, vemos que otra característica de ella es ser un dominio de sí, o una forma de moderación, en dos niveles: 1 . un control de los apetitos y cierta prudencia con respecto a las relaciones interpersonales. Lo cual es coherente con la definición de sophrosýne utilizada en el Fedro que establece que esta es una relación de dominio que se da cuando el juicio acerca de lo que es lo mejor (cosa que también pone de presente la necesidad de un conocimiento moral) domina sobre el deseo del gozo (Fedro, 237e-338b); y 2. cierta modestia intelectual que surge del saber que no se posee conocimiento moral. Esto es coherente con la actitud tomada por Cármides, y alabada por Sócrates, al responder si se considera sensato o no, diciendo que "si, a mi vez, digo que sí [soy sensato], y me alabo a mí mismo, es muy probable que esto parezca algo insufrible” ( Cármides, 158d).

Habiendo establecido ciertas características de quien posea la sensatez, las cuales, a pesar de que tal vez no constituyen la totalidad de la definición de esta virtud, serán útiles para establecer si Sócrates es o no sensato, considero pertinente establecer cómo podrían obtenerse estas características constituyentes del ser sensato. Adicionalmente, sé que el decir que quien es sensato posee cierto conocimiento moral, pero también cierta modestia intelectual que surge del saber que no se posee conocimiento moral, parece algo contradictorio. En las siguientes secciones mostraré por qué esto no constituye una contradicción.

\section{2. ¿CóMO ALCANZAR LA SOPHROSÝNE?}

\section{1. ¿Cómo alcanzar la sophrosýne como dominio de sí?}

Como se vio, una característica de la sophrosýne tiene que ver con el dominio de sí en los dos niveles mencionados. Frente a esto, el Cármides no da muchas luces, por lo cual describiré partes del proceso 
mediante el cual Sócrates intenta iniciar a Alcibíades en la filosofía en el Alcibiades I, para de ahí extraer el método que permite llegar a la sophrosýne como dominio de sí.

El propósito de Sócrates en la primera parte del diálogo (103a-124b) es demostrarle a Alcibíades su ignorancia moral, la cual Alcibíades empezaba a reconocer en $118 \mathrm{~b}$ y $119 \mathrm{a}-\mathrm{b}$, pero reconoce del todo en $124 \mathrm{~b}$ después de un largo discurso en el que Sócrates sostiene que sus rivales extranjeros (los de Alcibíades) lo superan en riqueza, educación y linaje, pero su visión agrandada de sí mismo, junto con su ignorancia, le impiden ver esto. A partir de dicho discurso, Alcibíades acepta que debe reconocer sus debilidades y carencias para enmendarlas y lograr su objetivo de gobernar. Es decir, que debe alcanzar cierto tipo de conocimiento de sí presentado como la consciencia de sus condiciones (debilidades y fortalezas) (Alcibiades I, 120e-124e). Pues este tipo de conocimiento de sí ha de permitir el "ser mejor"; el diálogo nos dice que el ser mejores es permitido por el arte de saber lo que somos (Alcibiades I, 128e), y también nos dice que aquello que somos es nuestra alma ( Alcibiades I, 129e-130c). Así, Sócrates establece que "la sabiduría consiste en conocerse a sí mismo [...]" ( Alcibiades I, 131b). Y este conocimiento del alma como consciencia de las propias condiciones, lleva al mejoramiento en tanto que permite la modestia intelectual descrita como parte de la virtud de la sophrosýne.

Teniendo esto en cuenta, resta establecer la forma en que se alcanza dicho conocimiento que ha de permitir la sophrosýne como dominio de sí en un segundo nivel. Con este propósito, Sócrates introduce en 133b la metáfora del ojo, invitando a Alcibíades a imaginar que en vez de "conócete a ti mismo", el oráculo dijese "mírate a ti mismo". Para lograr este cometido, el ojo debería mirarse a sí mismo en el reflejo de otro ojo, de igual manera:

Si el alma está dispuesta a conocerse a sí misma, tiene que mirar un alma, y sobre todo a la parte del alma en la que reside su propia facultad, la sabiduría, o a cualquier otro objeto que se le parezca. ( Alcibiades I, 133b)

Esto indica que la forma de alcanzar el conocimiento, que permitirá el dominio de sí como modestia intelectual, es mediante una conversación con otra alma. Pero no cualquier tipo de conversación; se requiere que la conversación se dé con miras a alcanzar el conocimiento.

Habiendo establecido cómo alcanzar la sophrosýne como dominio de sí en un segundo nivel, debemos establecer cómo alcanzar el primer nivel del dominio de sí: la dominación del juicio acerca de lo mejor sobre el deseo del gozo. Sobre esto podemos decir que, si el individuo se identifica con el alma, y si, como lo sugiere el Fedro (236d-238c), el alma es deseo y razón, entonces para que haya un dominio de la razón sobre el deseo, debemos i) conocer nuestros deseos y ii) tener el conocimiento moral que caracteriza la sophrosýne. El cómo hacer esto será analizado a continuación, junto al cómo llegar al conocimiento de sí entendido como conocimiento del conocimiento.

\section{1. ¿Cómo alcanzar la sophrosýne como conocimiento de sí/ del conocimiento?}

Como hemos visto, otra característica de la sophrosýne es que quien la posea ha de tener cierto conocimiento de sí como conocedor de la sensatez y ha de saber lo que es la sensatez. Esto implica cierto conocimiento moral al ser la sensatez una virtud y por ende algo bueno. Dicho conocimiento moral también es necesario a la hora de alcanzar la sensatez como el primer nivel, o momento, del dominio de sí. Ahora bien, Rappe argumenta que el método para alcanzar el conocimiento moral requerido para la sophrosýne es el examen socrático (élenchos), en tanto que este supone un cambio fundamental en los valores del interlocutor a partir del conocimiento de sí. Esta última afirmación se ve sustentada por el hecho de que el élenchos requiere que el interlocutor hable de acuerdo a lo que realmente cree. ${ }^{8}$ (Rappe, 2013, p. 6).

Sócrates es consciente de que el élenchos ha de producir algún conocimiento moral, dado que, con respecto a este método, él mismo afirma: 
[...] voy por todas partes sin hacer otra cosa que intentar persuadirlos, a jóvenes y viejos, a no ocuparos ni de los cuerpos ni de los bienes antes que del alma, ni con tanto afán, a fin de que ésta sea lo mejor posible, diciéndoos: 'No sale de las riquezas la virtud para los hombres, sino de la virtud, las riquezas y todos los otros bienes [...] ( Apologia, 30a-b).

En este sentido, el élenchos "en vez de buscar hacia afuera los objetos de deseo o los frutos de la acción, pide del interlocutor que dirija su atención hacia adentro; hacia sus estados de conocimiento". (Rappe, 2013, p. 16). Asimismo, Rappe sostiene que el élenchos captura la manera en la que un individuo entiende su rol en la pólis y muestra la manera como realmente debería entenderlo. Esta es la manera en la que el conocimiento de sí resultante del élenchos difiere del conocimiento técnico, pues "[...] el élenchos señala la inconmensurabilidad entre las metas de un individuo, quien necesariamente debe escoger un bien relativo a su propia perspectiva, y la meta del conocedor de sí como conocedor, para quien Sócrates sugiere que existe un bien absoluto". (Rappe, 2013, pp. 18-19).

Por otra parte, la autora señala que cuando en el Cármides Sócrates intenta esbozar una imagen del conocedor de sí mismo, habla de la posibilidad de un conocimiento auto-reflexivo usando los ejemplos de la sensación, los apetitos, los deseos, el miedo, la voluntad, y la opinión ( Cármides, 167d-168a). Y afirma que "en esta lista están incluidos todos los posibles estados de la experiencia humana, por lo menos en la visión platónica”. (Rappe, 2013, p. 21). Teniendo esto en cuenta, se puede decir que todos los estados anteriormente listados pueden ser concebidos como objetos del conocimiento de sí. Entonces, el élenchos puede verse como un método en el que el interlocutor advierte las sensaciones, deseos, apetitos, miedos, opiniones etc. que tiene y a partir de esta comprensión puede examinarlos para librarse de aquellos que considere dañinos (Rappe, 2013, p. 21).

El problema radica en que tenemos una justificación de que el élenchos debe producir el conocimiento moral necesario para la sophrosýne como conocimiento del sí en tanto conocimiento del conocimiento, y para la sophrosýne como el dominio de sí como dominio del juicio sobre el deseo, mas no tenemos una respuesta a cómo lograr esto mediante el élenchos. De hecho, Rappe misma nos dice que tal vez este conocimiento moral no es más que la "comprensión de que la virtud no puede ser definida sino únicamente vivida" (Rappe, 2013, p. 17). Sin embargo, no debemos apresurarnos a concluir esto, pues contradeciría las conclusiones de la primera sección. Veamos entonces lo que Hadot tiene pare decirnos frente al élenchos.

En su libro ¿Qué es la filosofía antigua?. Hadot empieza reconociendo que la concepción que en la antigüedad se tenía de la filosofía ${ }^{9}$ va más allá de la simple teoría, pues implica un compromiso del filósofo con su discurso. Sin este compromiso, la filosofía se reduce a un discurso despersonalizado, abstracto, y sistemático; se limita al uso informativo del lenguaje, cosa que Hadot no está dispuesto a aceptar. Para Hadot, el verdadero discurso filosófico, del cual el élenchos es la representación paradigmática, representa un uso del lenguaje que él denomina psicagógico; un uso del lenguaje mediante el cual las palabras transforman al hablante. Este uso del lenguaje se puede evidenciar al entender el discurso filosófico como un ejercicio espiritual.

Un ejercicio espiritual consiste en una transformación que inicia con un desprendimiento de la forma de vida que se solía llevar, y culmina con una inversión de valores. Si se entiende la filosofía como un ejercicio espiritual, entonces esta es una transformación de una vida no filosófica a una vida filosófica; es una elección vital como reacción crítica a la forma no filosófica de vivir, un cambio en lo que se tiene por bien o fin último, que lleva a un cambio a la hora de determinar cómo se valoran las cosas y cómo se encausan las acciones (Hadot, 1987, pp. 46-86; 104-114).

Independientemente del tema tratado, los diálogos con Sócrates remiten a la pregunta acerca de cómo vivir, ${ }^{10}$ asumiendo que toda acción de la vida humana se lleva a cabo con vistas al bien. Es decir que el examen socrático como ejercicio espiritual busca identificar qué es el bien. Esto se hace buscando fallas en la argumentación del interlocutor para de esta manera asegurar la coherencia. Dicha coherencia se da en varios niveles: 1. coherencia entre lo que se dice y lo que se piensa; 2 . coherencia entre lo que se hace y lo que se dice, y 3. coherencia con la verdad. 
A pesar de esto, a través de los diálogos, siendo el Cármides un ejemplo de esto, Sócrates y sus interlocutores fallan en definir las virtudes en cuestión. Esta incapacidad parece darse a causa del hecho que Sócrates dice no poseer ningún conocimiento conceptual (de la definición) de las virtudes, entonces el peso de la definición cae en el interlocutor quien suele estar confundido acerca de las virtudes. Sócrates entonces logra, sin definir cada virtud, disipar la confusión para responder al cómo vivir mediante una definición negativa de las virtudes, en tanto que no se sabe lo que son, pero se sabe lo que no son.

\subsection{Conclusiones acerca de cómo alcanzar la sophrosýne}

A la luz del ejercicio que Sócrates lleva a cabo con Alcibíades en el Alcibiades I, podemos establecer que la sophrosýne como dominio de sí en tanto modestia intelectual resultante del saber que no se tiene conocimiento moral, se alcanza mediante la conversación con otra alma con miras a alcanzar conclusiones racionales.

Por otro lado, a partir de las reflexiones de Rappe y Hadot, podemos decir que la sophrosýne como conocimiento de sí en tanto conocedor, y la sophrosýne como dominio de sí entendido como dominio del juicio sobre los deseos, parece alcanzarse mediante el élenchos. Pues i) este método supone un cambio en los valores del interlocutor; en tanto ejercicio espiritual, el élenchos supone un cambio en lo que se tiene por bien último. Esto es logrado mediante una definición negativa de las virtudes. Y ii) el élenchos permite al interlocutor darse cuenta de las sensaciones, deseos, apetitos, miedos, opiniones etc. que tiene, para de esta manera dominarlos a partir de la definición negativa de lo que no es la virtud. Esto constituye un primer paso para alcanzar la sophrosýne entendida como dominio del juicio sobre el deseo.

En cuanto al segundo paso para alcanzar la sophrosýne como dominio del juicio sobre el deseo, y en cuanto a la manera de alcanzar la sophrosýne como conocimiento de sí en tanto conocedor, sabemos que esto se logra mediante un conocimiento moral. Sin embargo, nada nos dice hasta ahora exactamente cómo se puede alcanzar este conocimiento moral, aunque tenemos claro que, para Sócrates, el élenchos es la manera, pues en cada una de sus conversaciones continúa indagando por la definición de las virtudes. Además, una de las conclusiones a las que se llega en el Cármides es que los buenos discursos, en este caso el élenchos (representados como metáfora en el ensalmo), llevan a la sensatez.( Cármides, 156b)

Ahora bien, antes de establecer si Sócrates se presenta o no como sensato, debemos conciliar la contradicción encontrada frente al hecho de que quien es sensato posee cierto conocimiento moral, pero también cierta modestia intelectual que surge del saber que no se posee conocimiento moral.

Hemos visto que la sophrosýne es una virtud con bastantes características que se pueden dar unas sin las otras pues, por ejemplo, la forma de alcanzar la modestia intelectual característica de la sophrosýne es mediante el reconocimiento de la ignorancia a partir de la conversación con otro (para este propósito no se requiere necesariamente de un conocimiento moral). Adicionalmente, en cuanto al dominio del juicio sobre los deseos hemos visto que hay dos pasos a dar (reconocer los deseos, y adquirir el conocimiento moral que permita dominarlos), de los cuales el primero se puede dar sin el segundo, pues el primero se da necesariamente en el élenchos, mientras que el segundo parece no darse a pesar de que Sócrates sostiene que este debería resultar del élenchos. De esta manera, dado que se pueden dar ciertas características de la sensatez sin otras, podemos decir que quien posea algunas características de ella, será más sensato que quien no posea ninguna. En este sentido, debemos reformular la modestia intelectual como característica de la sophrosýne, diciendo que quien no posea el conocimiento moral característico de la sophrosýne y necesario para alcanzar tanto el dominio del juicio sobre los deseos como el conocimiento de sí en tanto conocedor, pero demuestre cierta modestia intelectual como resultado del no poseer conocimiento moral, será más sensato que quien no muestre dicha modestia. Esto se debe a que, aunque no se conocerá a sí mismo en tanto conocedor de la sensatez, se conocerá a sí mismo en tanto no conocedor de la sensatez. 


\section{3. ¿Es Sócrates SEnSATo?}

Finalmente tenemos el material suficiente para evaluar si Sócrates es o no sensato. En primer lugar, es claro que Sócrates no posee el conocimiento moral que él mismo admite como necesario para la sophrosýne, esto es evidente i) por su famosa profesión de ignorancia a través de los diferentes diálogos, ii) por el hecho de que en ningún diálogo logra hallar una definición positiva de las virtudes que examina y iii) porque, como él señala en el Cármides:

Estás pensando que, por refutarte a ti mismo realmente, yo lo hago por otra causa distinta de aquella que me lleva a investigarme a mí mismo y a lo que digo, por temor tal vez a que se me escape el que pienso que sé algo, sin saberlo. ( Cármides, $166 \mathrm{~d})^{11}$

Entonces, si Sócrates ve la necesidad de investigarse/ examinarse a sí mismo con respecto al tema de la sensatez, es porque sabe que él mismo no la posee, pues si la poseyese, no creería que debe examinarse, ya que, como vimos, Sócrates sostiene que es el élenchos lo que lleva a poder definir las virtudes.

Por otro lado, debido a que Sócrates no posee el conocimiento de qué es la sensatez, no puede tampoco conocerse a sí mismo como conocedor de la sensatez, pues no es un conocedor de la sensatez, entonces no hay forma de que pueda conocerse como tal. Así, es claro que Sócrates no posee las dos primeras características señaladas como propias del sensato.

Sin embargo, como lo demuestran su profesión de ignorancia y el pasaje del Cármides anteriormente citado, Sócrates refleja la modestia intelectual que ha de surgir en alguien con cierto grado de sensatez si sabe que no posee conocimiento moral alguno. El reconocer esta falta de conocimiento moral en medio del diálogo con otro, demuestra que Sócrates se conoce a sí mismo en tanto no conocedor de la virtud. Haciendo que se pueda decir de él que, sin ser completamente sensato, es más sensato que sus conciudadanos quienes, como se hace explícito en La Apología (20c-24e), no reconocen su ignorancia en temas morales; no se conocen a sí mismos en tanto no conocedores de la virtud.

Adicionalmente, frente al dominio de sí entendido como dominio del juicio acerca de lo mejor sobre el deseo del gozo, Sócrates cumple parcialmente esta característica de la sophrosýne. Esto se debe a que, mediante el élenchos, Sócrates ha logrado reconocer sus deseos, al igual que sus opiniones y demás estados ya mencionados. A partir de esto, ha podido controlarlos como nos lo cuenta, por ejemplo, Alcibíades en el Banqueteal relatar i) que Sócrates no sucumbió ante los avances de Alcibíades al considerar que estos no llevarían a ningún beneficio mutuo ( Banquete, 216c-219a) y ii) que en la toma de Potidea, Sócrates controló su hambre y demás necesidades biológicas mejor que cualquier otro al saber que no había forma alguna de satisfacerlas ( Banquete, 219e-221c). Sin embargo, este control es posible para Sócrates a partir de la definición negativa de las virtudes que se alcanza mediante el élenchos, no a partir de un verdadero conocimiento moral, por lo tanto, existe la posibilidad de que el juicio acerca de lo mejor que se da en Sócrates, falle.

En suma, Sócrates no puede ser llamado completamente sensato al no poder definir la sensatez y, por ende, no poderse saber a sí mismo como sensato. No obstante, es más sensato que sus conciudadanos i) al reconocer su ignorancia frente a la sensatez y mostrar cierta modestia intelectual y ii) dominar sus deseos a partir del mejor juicio sobre lo mejor que logra obtener mediante la definición negativa de las virtudes. Sócrates parece ser entonces un caso de alguien sensato que no sabe que lo es, sin embargo, como se mostró tanto en el Cármides como en el presente trabajo, dicho caso no se puede dar. En este sentido, cabe tener en cuenta una afirmación ya citada al respecto:

"Parece que, quien algunas veces obra con provecho, obra, en verdad, con sensatez y es sensato, aunque no sabe que lo es" ( Cármides, 164c), dice Sócrates antes de que Critias refute esta posibilidad. No obstante, esta afirmación suscita la pregunta acerca de si el ser sensato es lo mismo que el obrar con sensatez. Como ya vimos, existe una diferencia entre estas dos cosas, pues el obrar con sensatez radica en el saber lo que uno 
está haciendo, mientras que el ser sensato radica en el saber que se es sensato al saber que se está obrando con sensatez. De esta manera, podemos concluir que, aunque Sócrates no es completamente sensato, suele obrar con sensatez.

Ahora bien, habiendo alcanzado las conclusiones pertinentes con respecto a la sensatez de Sócrates, encuentro sumamente desconcertante el hecho que Sócrates no logre obtener el conocimiento moral que lo haría sensato al saberse sensato, a pesar de que se encuentra convencido de que dicho conocimiento moral puede ser alcanzado mediante el élenchos. A partir de este desconcierto, quisiera a continuación intentar señalar qué falla en el élenchos impidiendo a Sócrates alcanzar la sensatez.

\section{APÉNDICE: ¿QuÉ FALlas haY EN EL Élenchos QUE IMPIDEN a Sócrates alcanzar la SOPHROSÝNE?}

Frente a la pregunta acerca de por qué falla Sócrates en alcanzar la sensatez, McKim nos dice:

Platón presenta el auto-conocimiento socrático como insuficiente frente al conocimiento de lo que es la virtud. También presenta el método socrático como incapaz de subsanar dicha insuficiencia. (McKim, 2017, p. 60)

Y argumenta que el porqué de esto se puede encontrar analizando la segunda parte del Cármides, pues "la téchne que el conocedor del conocimiento del que habla Critias usaría para probar a los que dicen poseer conocimiento, se puede identificar con el élenchos". (McKim, 2017, p. 63)

Ahora bien, el problema con este método es que las conclusiones que de él se extraen acerca de lo que no son las virtudes, surgen del rechazo de alguna tesis por ser contradictoria con el conjunto de creencias de cada interlocutor. Esto lleva a asumir cosas como que toda virtud es buena y útil, sin que este tipo de afirmaciones sean probadas. ${ }^{12}$ Por eso, en los casos en los que Sócrates se encuentra frente a interlocutores que no sostienen que la virtud es buena (Calicles en el Gorgias y Trasímaco en la República), debe convencerlos de que realmente no creen esto, todo con el fin de refutar sus definiciones de las virtudes pertinentes en cada caso, a partir de la coherencia con un sistema de creencias compartido por Sócrates y sus interlocutores. En este sentido:

$\mathrm{Al}$ enfrentarse a un interlocutor que sostenga que sabe que la virtud es algo diferente al conocimiento del bien y del mal, Sócrates puede mostrarle que esa afirmación es inconsistente con su creencia común de que la virtud siempre es útil. (McKim, 2017,72)

Adicionalmente, Sócrates sostiene lo que McKim llama el principio "ti-antes-que-poîon": sostiene que no podemos tener conocimiento acerca de la clase de cosa (poîn) que algo es, sin antes saber qué ( $t i$ ) es dicho algo. En este sentido, al Sócrates iniciar sus búsquedas acerca de lo que son las virtudes a partir del presupuesto de que ellas son buenas/ útiles, rompe dicho principio, pues la bondad y la utilidad señalan cómo es la cosa en cuestión (poîn) -en este caso las virtudes- antes de saber lo que ellas son ( $t i \hat{)}$. Es por esto que el presupuesto socrático de la bondad/ utilidad de las virtudes, no es un verdadero conocimiento, sino que se queda en la mera opinión (dóxa). Y si Sócrates inicia el élenchos a partir de este supuesto, no podrá generar más que opiniones sobre la virtud, al partir precisamente de una opinión. Esto se hace evidente desde el principio del Cármides, pues al dirigirse a Cármides, Sócrates dice que "es manifiesto que, si la sensatez es algo tuyo, tendrás una cierta opinión de ella [...]” ( Cármides, 159a). Utilizando la palabra dóxa (“opinión”), en lugar de la palabra epistéme ("conocimiento"):

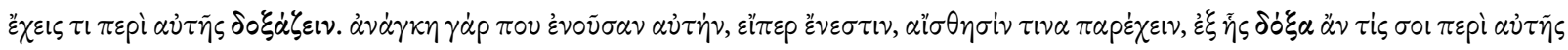

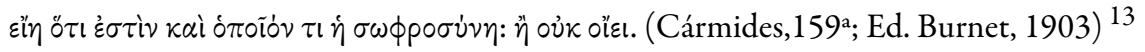

Pues, forzosamente, estando en ti, en el supuesto de que lo esté, te suministrará una cierta sensación de la que se alce alguna posible opinión sobre ella: o sea, sobre qué es y cuáles son las propiedades de la sensatez. ¿No te parece? 
Pues, forzosamente, estando en ti, en el supuesto de que lo esté, te suministrará una cierta sensación de la que se alce alguna posible opinión sobre ella: o sea, sobre qué es y cuáles son las propiedades de la sensatez. ¿No te parece?

En suma, las razones por las cuales el élenchos falla en alcanzar el conocimiento moral que habría de permitir la sophrosýne son i) que a pesar de que el conocimiento moral parece ser algo universal para Sócrates, el examen que pretende llegar a este llega a conclusiones a partir de la coherencia de una red de creencias o individual o particular. ${ }^{14}$ Y ii) que asumiendo que las virtudes son buenas y útiles sin antes saber lo que son las virtudes, Sócrates pretende alcanzar el conocimiento verdadero a partir de una mera opinión, lo cual, en términos lógicos, no es posible.

\section{REFERENCIAS}

Burnet, J. (1903). Plato. Platonis Opera. Oxford: Oxford University Press.

Hadot, P. (1987). Exercices spirituels et philosophie antique. Paris: Etudes augustiniennes.

Hadot, P. (1998). ¿Qué es la filosofía antigua? México: Fondo de cultura económica.

Kosman, L. A. (2014). Self-Knowledge and Self-Control in Plato's Charmides. UC Denver Health SC lib. Recuperado de https://dspace.library.uu.nl/bitstream/handle/1874/372518/RMAPhilosophyThesis-AleksanderBrodowic z.pdf?sequence $=2 \&$ isAllowed $=\mathrm{y}$

McKim, R. (2017). Socratic Self-Knowledge and "Knowledge of Knowledge" in Plato's Charmides. Baltimore: The Johns Hopkins University Press.

Plato (2008). Charmides. En The Dialogues of Plato. Benjamin Jowett (trad). Lugar: The Project Gutenberg (eBook). Recuperado de https://www.gutenberg.org/files/1580/1580-h/1580-h.htm

Platón (1992). Alcibíades I. En Diálogos VII.Juan Zaragoza y Pilar Gómez Cardó (trads) (pp.16-86). Madrid: Editorial Gredos.

Platón (1985). Apología de Sócrates. En Diálogos I. J. Calonge Ruiz, E. Lledó Iñigo, C. García Gual (trads.) (pp. 148186). Madrid: Editorial Gredos.

Platón (1988). Banquete. En Diálogos III. C. García Gual, M. Martínez Hernández, E. Lledó Íñigo (trads.) (pp. 145284). Madrid: Editorial Gredos.

Platón (1985). Cármides. En Diálogos I. J. Calonge Ruiz, E. Lledó Iñigo, C. García Gual (trads.) (pp. 326- 368). Madrid: Editorial Gredos.

Platon (2015). "Charmide” dans artyuiop. Émile Chambry (trad). France. http://www.artyuiop.fr/artyuiop/Platon_ -_Charmide_files/artyuiop94-Platon-Charmide-trad._Emile_Chambry.pdf

Platón (2005). Charmides. En Platon: die Werke vollständig in deutscher Sprache. Rudolf Haller (trad.) Deutschland. Recuperado de http://www.opera-platonis.de/Platon_Werke.pdf

Platón (1988). Fedro. En Diálogos III. C. García Gual, M. Martínez Hernández, E. Lledó Íñigo (trads.) (pp. 284- 413). Madrid: Editorial Gredos.

Platón (1987). Gorgias. En Diálogos II. J. Calonge Ruiz, E. Acosta Méndez, F. J Olivieri, J. L Calvo (trads.) (pp. 8145). Madrid: Editorial Gredos.

Platón (1985). Laques. En Diálogos I. J. Calonge Ruiz, E. Lledó Íñigo, C. García Gual (trads.) (pp. 443-486). Madrid: Editorial Gredos.

Platón (1871). Protágoras. En Obras completas de Platón (pp. 15-92). Madrid: Medin y Navarro.

Platón (1988). República. En Diálogos $I V$. Conrado Eggers Lan (trad.). Madrid: Editorial Gredos.

Rappe, S. (2013) Socrates and Self-knowledge. Univeristy of South Carolina.

Schmid, W. (1983). Socratic Moderation and Self-Knowledge. Journal of the History of Philosophy 21(3), 339- 348.

Smith, N. (2004). Did Plato Write the Alicibiades I? Apeiron 37 (2): 93- 108. 


\section{Notas}

1 Cabe señalar que desde la antigüedad hasta nuestros días existe cierta polémica acerca de la autenticidad de este diálogo, el cual fue descrito por Jámblico como conteniendo toda la sabiduría platónica, mientras que Aristóteles, la autoridad más confiable acerca del trabajo platónico, ni siquiera menciona la existencia del diálogo. Igualmente, autores contemporáneos como Julia Annas y Paul Friedländer defienden la autenticidad del Alcibiades I, mientras que otros como Leonard Brandwood y Pamela Clark la refutan (Smith, 2004, pp. 92-95).

2 Todas las traducciones del inglés son mías a menos de que se indique lo contrario.

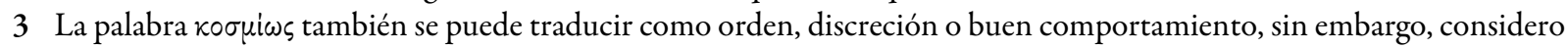
que todas estas traducciones se ven abarcadas en el concepto de "moderación”.

4 Énfasis agregados. Las traducciones del diálogo se ajustan a la edición de Calonge Ruiz (1985).

5 Establecida en Grecia alrededor del 1200 a.C.

6 De Sócrates.

7 Pues, como ya se ha visto, el dominio de sí parece ser sólo una parte de la sophrosýne.

8 Esta condición como requerimiento para el diálogo se hace explícita en diferentes diálogos, por ejemplo, en el Gorgias (495a).

9 Concepción que él busca rescatar.

10 Creo que esta afirmación no es difícil de aceptar si se ha leído La Apología y/ o la descripción que Nicias hace en el Laques de la conversación con Sócrates ( Laques, 187e-188a).

11 Sócrates dirigiéndose a Critias.

12 Cabe aclarar que no estoy queriendo señalar que las virtudes no sean buenas o bellas, sino que para que el examen socrático pueda cumplir su propósito, debe probar que las virtudes son buenas/ bellas y partir de allí, no asumir que lo son sin prueba alguna.

13. Los énfasis son míos.

14 Por "particular" entiendo "perteneciente a una comunidad específica".

\section{BY-NC-SA}

